\title{
1 kHz, multi-mJ Yb:KYW bulk regenerative amplifier
}

\author{
Anne-Laure Calendron ${ }^{1,2 *}$, Hüseyin Çankaya ${ }^{1,2}$ and Franz X. Kärtner ${ }^{1,2,3}$ \\ ${ }^{\prime}$ Center for Free-Electron Laser Science, Deutsches Elektronen Synchrotron, Notkestrasse 85, 22607 Hamburg, Germany \\ ${ }^{2}$ The Hamburg Centre for Ultrafast Imaging, Universität Hamburg, Luruper Chaussee 149, 22761 Hamburg, Germany \\ ${ }^{3}$ Department of Electrical Engineering and Computer Science and Research Laboratory of Electronics, \\ Massachusetts Institute of Technology, Cambridge, Massachusetts 02139, USA \\ "Corresponding Author, e-mail: anne-laure.calendron@desy.de
}

\begin{abstract}
We report on a high-energy Yb:KYW dual-crystal regenerative amplifier, delivering $6 \mathrm{~mJ}$ at $1 \mathrm{kHz}$. The pulses centered at $1030 \mathrm{~nm}$, stretched with chirped fiber Bragg gratings, can sustain a sub-ps duration after recompression.

OCIS codes: (140.3280) Laser amplifiers; (140.3538) Lasers pulsed; (140.3615) Lasers, ytterbium
\end{abstract}

\section{Introduction}

High energy lasers are sought for pumping optical parametric chirped pulse amplifiers (OPCPA), generating ultrashort pulses suitable for the creation of single attosecond pulses via high harmonic generation (HHG).

Ytterbium doped laser media are attractive for high energy pulses due to low quantum defect, lack of parasitic effects such as excited-state absorption, cross relaxation and up-conversion, leading to high efficiency and low heat load operation. In addition, most Ytterbium-doped materials provide emission linewidths that allow for fs-pulse generation. Among the well-developed, hence reliable materials, Yb:KYW has a thermal conductivity of $3.6 \mathrm{~W} / \mathrm{m} / \mathrm{K}$, a lifetime of $300 \mu \mathrm{s}$ [1] and its emission spectrum supports pulses a few hundreds of femtosecond long. Our pump line for OPCPAs is constituted of a regenerative amplifier followed by a booster amplifier based on cryogenically cooled $\mathrm{Yb}: \mathrm{YAG}$. Yb:KYW is suitable gain medium for the first amplification stage in the pump chain because the peak wavelength corresponds to $\mathrm{Yb}: \mathrm{YAG}$ emission peak and it provides enough bandwidth supporting sub-ps pulse duration to pump the first OPA stages.

Previously, high energy $\mathrm{Yb}: \mathrm{KYW}$ based regenerative amplifiers were demonstrated as thin-disc, as in Ref [2]. In 2007, a cryogenic Yb:KYW laser was reported with $5.5 \mathrm{~mJ}$ energy and $3.4 \mathrm{~nm}$ wide spectrum, enhanced by shaping the seed spectrum [3]. In addition, the multi-pass Yb:KYW amplifier scheme delivering $27 \mathrm{~mJ} \mathrm{at} 100 \mathrm{~Hz}$ was demonstrated [4] in 2011. In this work, we describe and demonstrate a Yb:KYW bulk regenerative amplifier reaching to $6.5 \mathrm{~mJ}$ at room temperature, with 70 decades gain.

\section{Experimental}

The experimental set-up, shown in Figure 1, was constituted of a seeder, a stretcher and a regenerative amplifier followed by a grating compressor. The commercial, $42 \mathrm{MHz} \mathrm{Yb}: \mathrm{KYW}$ oscillator delivered transform limited $210 \mathrm{fs}$ pulses centered at $1030 \mathrm{~nm}$ wavelength. The stretcher consisted of four chirped-fiber Bragg gratings (CFBG1-4), whose dispersion is matched to the grating compressor. The stretching ratio was chosen considering the complete amplification chain, inclusive a future booster after the regenerative amplifier. To avoid non-linearities in the fibers, the seed output was attenuated to the $\mathrm{mW}$ level, but needed to be amplified in between the CFBG's to also compensate for the losses introduced by the circulators (C1-3). At the output of the stretcher including the fiber amplifiers (FA1-2), pulses with $0.6 \mathrm{~nJ}$ energy and $3.2 \mathrm{~ns}$ duration were send into the regenerative amplifier. The latter relied on a two-ytterbium doped KYW crystal resonator with a similar configuration to the one described in [5]. The short cavity, symmetric on S, middle point between both crystals, and containing both crystals and mirrors M1, M2 and DC, was extended with a 4-f telescope to integrate the switching elements (Pockels cell, thin-film polarizer and quarter-waveplate). In order to minimize the non-linearities, hence the aberrations on the beam, the spot sizes were increased up to $400 \mu \mathrm{m}$ in the crystals and $1 \mathrm{~mm}$ in the switching elements. The compressor is based on two multi-dielectric gratings with 1760 lines $/ \mathrm{mm}$ at $60^{\circ}$ angle of incidence. 


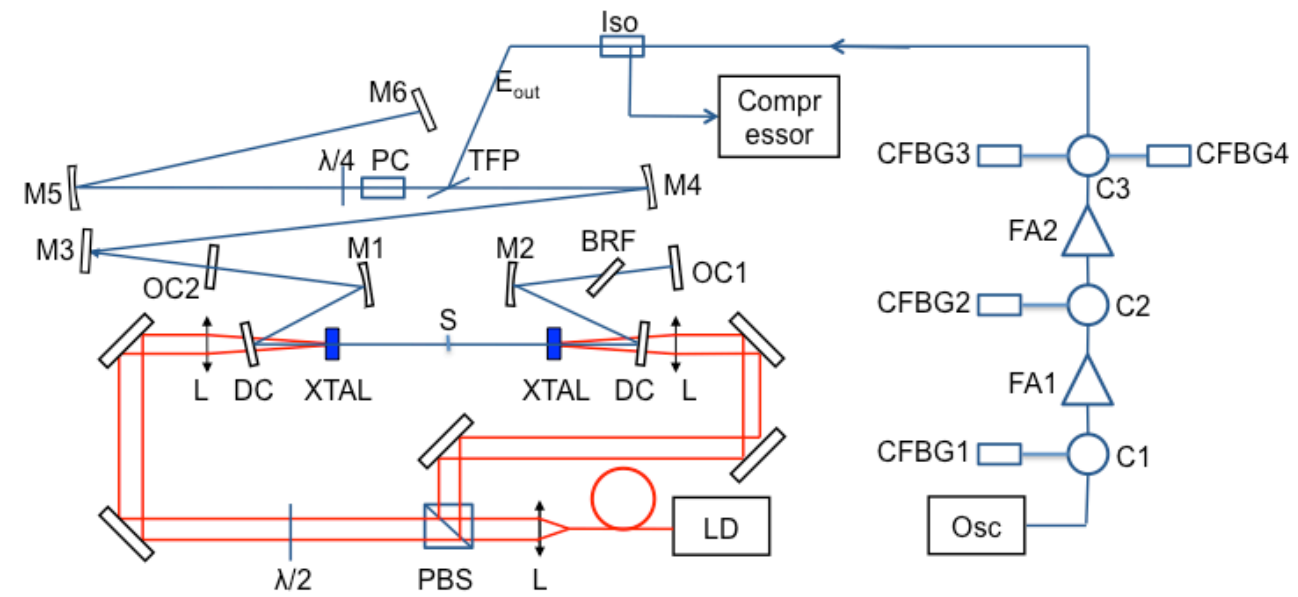

Fig. 1. Layout of the regenerative amplifier with seeder and stretcher. The input in the regenerative amplifier consisted of oscillator (Osc), chirped fiber Bragg gratings (CFBG1-4), 3-port circulators (C1,C2), 4-port circulator (C3), fiber amplifiers (FA1-2), a Faraday isolator (Iso). The cavity of the regenerative amplifier included both crystals (XTAL's), dichroic mirrors (DC's), Pockels cell (PC), thin film polarizer (TFP), quarter-waveplate ( $\lambda / 4)$, high reflective mirrors (M1-M6), where M1 and M2 are curved mirrors with $500 \mathrm{~mm}$ radius of curvature, and M4 and M5 have $1000 \mathrm{~mm}$ radius of curvature. To acquire the tuning curve, a birefringent filter (BRF) was inserted in the cavity. For testing the laser head in $\mathrm{CW}$ operation, the output couplers $\mathrm{OC} 1$ and $\mathrm{OC} 2$ were inserted; $\mathrm{OC} 1$ was replaced with a high-reflector for operation as a regenerative amplifier.

The $2 \%$ doped, $3 \mathrm{~mm}$ long crystals cut along the $\mathrm{n}_{\mathrm{g}}$ axis were pumped with a fiber-coupled laser diode, delivering a maximum of $120 \mathrm{~W}$ at a wavelength of $981 \mathrm{~nm}$, corresponding to the peak absorption of the material. The horizontal polarization of the amplified field corresponded to the $\mathrm{n}_{\mathrm{m}}$ axis of the crystal in order to match later on the wavelength of the following amplifier, centered at $1030 \mathrm{~nm}$. It also consequently allowed cooling along the $\mathrm{n}_{\mathrm{p}}$ axis of the crystal, which is of advantage for its higher thermal conductivity coefficient.

\section{Results}

The laser-head was first characterized as a continuous-wave oscillator. Without switching elements but inserting a $15 \%$ output coupler, the slope efficiency was measured to be $40 \%$. The maximum output power extractable with a beam quality factor better than 1.1 was $19.4 \mathrm{~W}$. The tuning curve, measured by inserting a birefringent filter in the cavity and for $15 \%$ output coupling, illustrates the tunability between $1021 \mathrm{~nm}$ and $1033 \mathrm{~nm}$, with a peak output power at $1031 \mathrm{~nm}$, as displayed on Fig. 2 (left). The beam quality, measured with the 4-sigma algorithm, was equally good $\left(\mathrm{M}^{2}<1.1\right)$ when operating as regenerative amplifier, as shown on Fig. 2 (right).
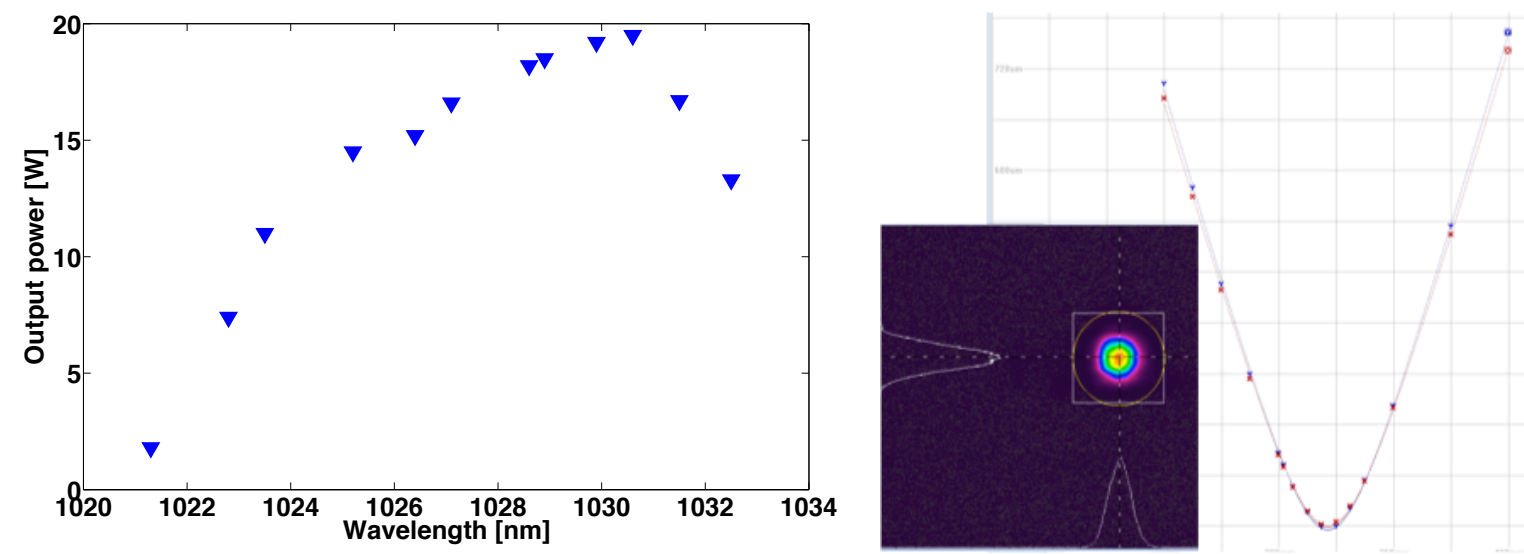

Fig. 2. Characterization of the laser-head: left, tuning curve taken in cw operation, and right, measurement of the beam quality in regenerative operation.

Figure 3 (left) shows the efficiency curve during the cavity-dumped operation of the laser. In this operation regime, the output energy was $5.5 \mathrm{~mJ}$ for $130 \mathrm{~W}$ pump power, $825 \mathrm{~ns}$ extraction time and $500 \mu$ s pumping duration at $1 \mathrm{kHz}$. Figure 3 (right) compares the spectra from the seeder and at the output of the amplifier, operating first in cavity-dumping and second as regenerative amplifier. The seed spectrum was centered around $\mathrm{nm}$ and $4.9 \mathrm{~nm}$ wide. 
The structures on it were due to self-phase modulation in the fiber amplifiers and the sharp edges to the filtering in the CFBGs. The lower part overlapped with the $5.7 \mathrm{~nm}$ wide spectrum of the resonator in cavity-dumped operation. As a result, the seed spectra was reshaped and narrowed to $3.6 \mathrm{~nm}$, which supports Fourier-transform limited pulse duration below $400 \mathrm{fs}$. Due to the gain narrowing, the pulses were shortened to $2.35 \mathrm{~ns}$ at the output.
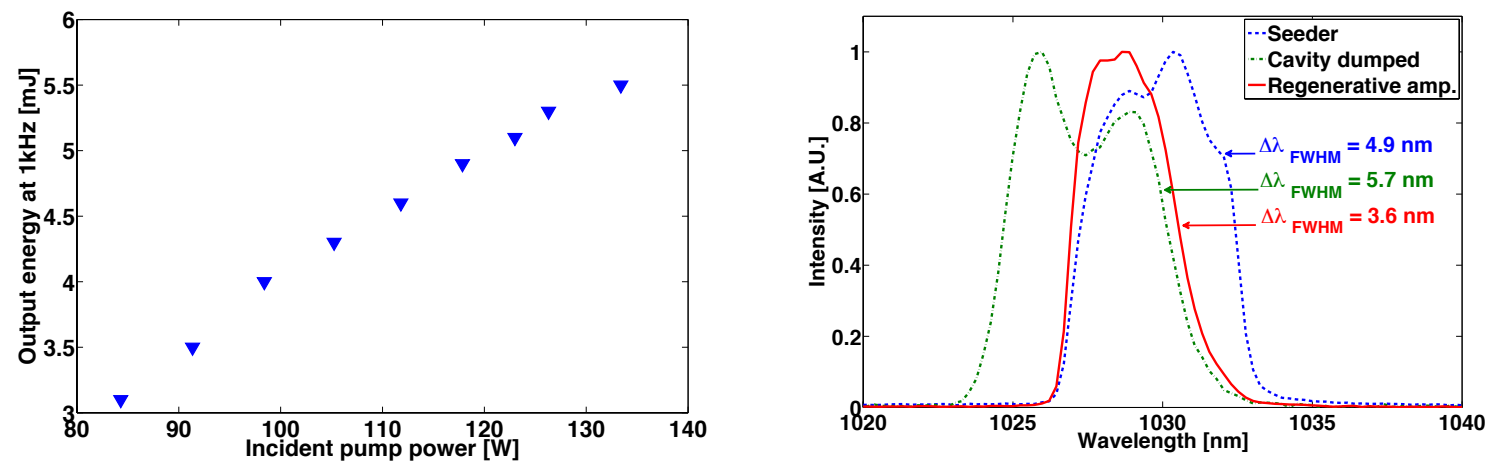

Fig. 3. Spectra of the seeder (blue, dashed curve), the laser-head in cavity-dumped (green, dot-dashed curve) and regenerative amplifier (red, plain curve) operation.

As a regenerative amplifier, $6.5 \mathrm{~mJ}$ were extracted after $600 \mathrm{~ns}$ extraction time, corresponding to 30 round-trips, for $500 \mu \mathrm{s}$ pumping time at full pump power. A measurement of the pulse energy over $60 \mathrm{~h}$, recording every pulse, showed a standard deviation of less than $100 \mu \mathrm{J}$. The pre- and post-pulses were measured to be smaller than $1 \%$ and $1.5 \%$, respectively.

This is to date, to our knowledge, the highest energy demonstrated with a bulk Yb:KYW regenerative amplifier.

\section{Conclusion}

In summary, we demonstrated a dual-slab Yb:KYW regenerative amplifier, delivering $6 \mathrm{~mJ}$ at $1 \mathrm{kHz}$. Even after gain narrowing, the spectrum supports sub-ps long pulses. This will later be further amplified to reach higher energy.

\section{References}

[1] K. Petermann, D. Fagundes-Peters, J. Johannsen, M. Mond, V. Peters, J.J. Romero, S. Kutovoi, J. Speiser, A. Giesen, "Highly Yb-doped oxides for thin-disc lasers," J. of Crystal Growth, 275 (1-2), 135-140 (2005).

[2] M. Delaigue, I. Manek-Hönninger, C. Hönninger, A. Courjaud, and E. Mottay, “1 mJ, Multi-kHz, Sub-500 fs Diode-Pumped Ytterbium Laser Amplifier," CLEO, Baltimore (2007)

[3] K. Ogawa, Y. Akahane, M. Aoyama, K. Tsuji, S. Tokita, J. Kawanaka, H. Nishioka, and K. Yamakawa, "Multi-millijoule, diode-pumped, cryogenically-cooled $\mathrm{Yb}: \mathrm{KY}\left(\mathrm{WO}_{4}\right)_{2}$ chirped-pulse regenerative amplifier," Opt. Expr., 15 (14), 8598-8602 (2007)

[4] D.N. Papadopoulos, A. Pellegrina, L.P. Ramirez, P. Georges, and F. Druon, "Broadband high-energy diode-pumped Yb:KYW multipass amplifier," Opt. Lett., 36 (19), 3816-3818 (2011)

[5] A.-L. Calendron, “Dual-crystal Yb:CALGO high power laser and regenerative amplifier,” Opt. Expr., 21 (22), 26174-26181 (2013) 